\title{
Dynamic Reconfiguration of Mission Parameters in Underwater Human-Robot Collaboration
}

\author{
Md Jahidul Islam ${ }^{1}$, Marc $\mathrm{Ho}^{2}$, and Junaed Sattar ${ }^{3}$
}

\begin{abstract}
This paper presents a real-time programming and parameter reconfiguration method for autonomous underwater robots in human-robot collaborative tasks. Using a set of intuitive and meaningful hand gestures, we develop a syntactically simple framework that is computationally more efficient than a complex, grammar-based approach. In the proposed framework, a convolutional neural network is trained to provide accurate hand gesture recognition; subsequently, a finite-state machine-based deterministic model performs efficient gestureto-instruction mapping and further improves robustness of the interaction scheme. The key aspect of this framework is that it can be easily adopted by divers for communicating simple instructions to underwater robots without using artificial tags such as fiducial markers or requiring memorization of a potentially complex set of language rules. Extensive experiments are performed both on field-trial data and through simulation, which demonstrate the robustness, efficiency, and portability of this framework in a number of different scenarios. Finally, a user interaction study is presented that illustrates the gain in the ease of use of our proposed interaction framework compared to the existing methods for the underwater domain.
\end{abstract}

\section{INTRODUCTION}

Underwater robotics is an area of significantly increasing importance and applications, and is experiencing a rapid rise in research endeavors. Truly autonomous underwater navigation is still an open problem, with the underwater domain posing unique challenges to robotic sensing, perception, navigation, and manipulation. However, a simple yet robust human-robot communication framework [4], [9], [23] is desired in many tasks which requires the use of autonomous underwater vehicles (AUVs). Particularly, the ability to accept direct human guidance and instructions during task execution (see Fig. 1) is of vital importance. Additionally, such semi-autonomous behavior of a mobile robot with human-in-the-loop guidance reduces operational overhead by eliminating the necessity of teleoperation (and one or more teleoperators). However, simple and intuitive instruction sets and robust instruction-to-action mapping are essential for successful use of AUVs in a number of critical applications such as search-and-rescue, surveillance, underwater infrastructure inspection, and marine ecosystem monitoring.

The ability to alter parts of instructions (i.e., modifying subtasks in a larger instruction set) and reconfigure program parameters is often important for underwater exploration and data collection processes. Because of the specific challenges

The authors are with the Department of Computer Science and Engineering, University of Minnesota-Twin Cities, MN 55455, USA.

E-mail: $\left\{{ }^{1}\right.$ islam034, ${ }^{2}$ hoxxx323, ${ }^{3}$ junaed $\} @$ umn.edu in the underwater domain, what would otherwise be straightforward deployments in terrestrial settings often become extremely complex undertakings for underwater robots, which require close human supervision. Since Wi-Fi or radio (i.e., electromagnetic) communication is not available or severely degraded underwater [7], such methods cannot be used to instruct an AUV to dynamically reconfigure command parameters. The current task thus needs to be interrupted, and the robot needs to be brought to the surface in order to reconfigure its parameters. This is inconvenient and often expensive in terms of time and physical resources. Therefore, triggering parameter changes based on human input while the robot is underwater, without requiring a trip to the surface, is a simpler and more efficient alternative approach.

Controlling a robot using speech, direct input (e.g., a keyboard or joystick), or free-form gestures is a general paradigm [3], [5], [22] in the context of Human-Robot Interaction (HRI). Unlike relatively less challenging terrestrial environments, the use of keyboard or joystick interfaces or tactile sensors is unappealing in underwater applications since it entails costly waterproofing and introduces an additional point of failure. Additionally, since speech or RGB$\mathrm{D}$ (i.e., visual and depth image)-based interfaces, such as a Leap Motion ${ }^{\mathrm{TM}}$ or Kinect ${ }^{\mathrm{TM}}$ are not feasible underwater, vision-based communication schemes are more natural for diver-robot interaction.

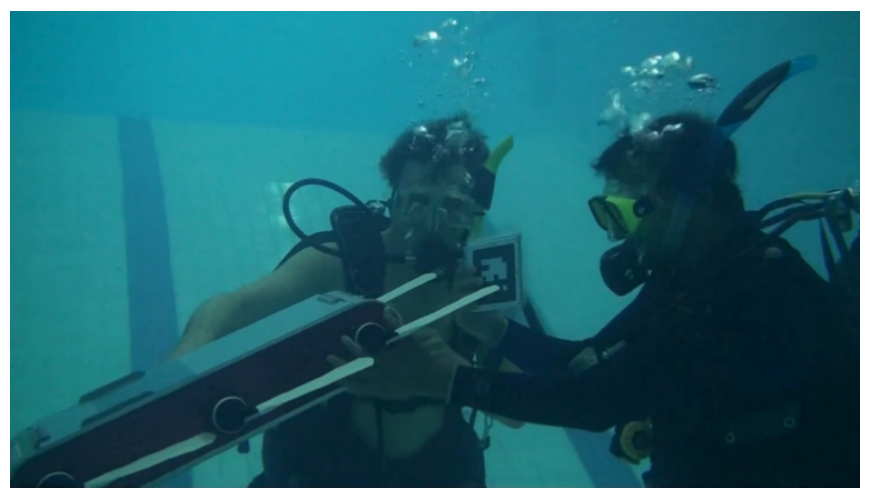

Fig. 1: Divers programming an AUV using the RoboChat [9] language using ARTag [11] markers; note the thick "tag book" being carried by the diver, which, while necessary, adds to the diver's cognitive load and impacts mission performance

This work explores the challenges involved in designing a hand gesture-based human-robot communication framework for underwater robots. In particular, a simple interaction 
framework is developed where a diver can use a set of intuitive and meaningful hand gestures to program the accompanying robot or reconfigure program parameters on the fly. A convolutional neural network-based robust hand gesture recognizer is used with a simple set of gesture-toinstruction mapping. A finite-state machine based interpreter ensures predictable robot behavior by eliminating spurious inputs and incorrect instruction compositions.

\section{RELATED WORK}

Modulating robot control based on human input in the form of speech, hand gestures, or keyboard interfaces has been explored extensively for terrestrial environments [3], [5], [20], [22]. However, most of these human-robot communication modules are not readily applicable in underwater applications due to environmental and operational constraints [7]. Since visual communication is a feasible and operationally simpler method, a number of visual diver-robot interaction frameworks have been developed in the literature.

A gesture-based framework for underwater visual-servo control was introduced in [8], where a human operator on the surface was required to interpret the gestures and modulate robot movements. Due to challenging visual conditions underwater [7] and lack of robust gesture recognition techniques, fiducial markers were used in lieu of free-form hand gestures as they are efficiently and robustly detectable under noisy conditions. In this regard, most commonly used fiducial markers have been those with square, black-andwhite patterns providing high contrast, such as ARTags [11] and April Tags [17], among others. These consist of black symbols on a white background (or the opposite) in different patterns enclosed within a square. Circular markers with similar patterns such as the Photomodeler Coded Targets Module system and Fourier Tags [18] have also been used in practice.

RoboChat [9] is the first visual language proposed for underwater diver-robot communication. Divers use a set of ARTag markers printed on cards to display predefined sequences of symbolic patterns to the robot, though the system is independent of the exact family of fiducial markers being used. These symbol sequences are mapped to commands using a set of grammar rules defined for the language. These grammar rules include both terse imperative action commands as well as complex procedural statements. Despite its utility, RoboChat suffers from two critical weaknesses. Firstly, because a separate marker is required for each token (i.e., a language component), a large number of marker cards need to be securely carried during the mission and divers have to search for the cards required to formulate a syntactically correct script; this whole process imposes a rather high cognitive load on the diver. Secondly, the symbolto-instruction mapping is inherently unintuitive, which makes it inconvenient for rapidly programming a robot. The first limitation is addressed in [23], where a set of discrete motions using a pair of fiducial markers is interpreted as a robot command. Different features such as shape, orientation, and size of these gestures are extracted from the observed motion and mapped to the robot instructions. Since more information is embeddable in each trajectory, a large number of instructions can be supported using only two fiducial markers. However, this method introduces additional computational overhead to track the marker motion and needs robust detection of shape, orientation, and size of the motion trajectory. Furthermore, these problems are exacerbated since both robot and human are suspended in a sixdegrees-of-freedom (6DOF) environment. Also, the symbolto-instruction mapping remains unintuitive.

Since the traditional method for communication between scuba divers is with hand gestures, similarly instructing robots is more intuitive and flexible than using fiducial markers. Additionally, it relieves the divers of the task of carrying a set of markers, which, if lost, would put the mission in peril. There exists a number of hand gesture-based HRI frameworks [3], [5], [21], [22] for terrestrial robots. In addition, recent visual hand gesture recognition techniques [13]-[15] based on convolutional neural networks have been shown to be highly accurate and robust to noise and visual distortions [10]. A number of such visual recognition and tracking techniques have been successfully used for underwater tracking [19] and have proven to be more robust than other purely feature-based methods (e.g., [12]). However, feasibility of these models for hand gesture based diver-robot communication has not been explored in-depth yet.

\section{Methodology}

The proposed framework is built on a number of components: the choice of hand gestures to map to command tokens, the robust recognition of hand gestures, and the use of a finite-state machine to enforce command structure and ignore erroneous detections or malformed commands. Each of these components is described in detail in the following sections.

\section{A. Mapping Hand Gestures to Language Tokens}

The key objective of this work is to design a simple, yet expressive framework that can be easily adopted by divers for communicating instructions to the robot without using fiducial markers or memorizing complex language rules. Therefore, we choose a small collection of visually distinctive and intuitive gestures, which would improve the likelihood of robust recognition in degraded visual conditions. Specifically, we use only the ten gestures shown in Fig. 2

As seen in Fig. 2, each gesture is intuitively associated with the command it delivers. Sequences of different combinations of these gestures formed with both hands are mapped to specific instructions. This work concentrates on two different sets of instructions as illustrated in Fig. 3 . which are in the following form:

- Task switching: This is for instructing the robot to stop executing the current program and start a task specified by the diver, such as hovering, following, or moving left/right/up/down, etc. In other words, these commands are atomic behaviors that the robot is capable 


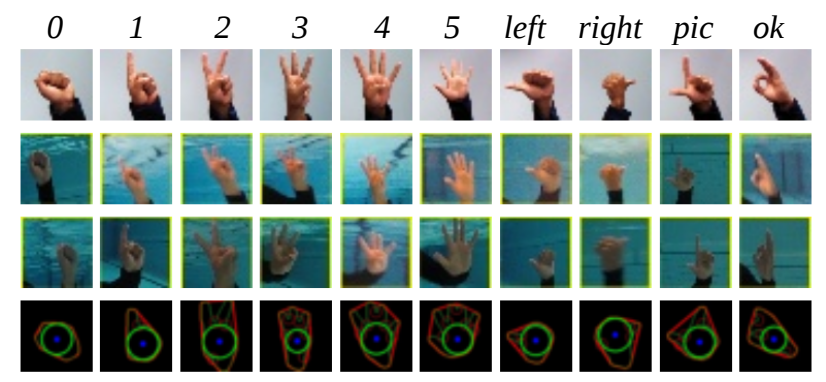

Fig. 2: Hand gestures used in the proposed work. The first three rows show sampled training images for ten different hand gestures in separate columns; the bottom row shows expected hand-contour with different curvature markers for each gesture.

of executing. An optional argument can be provided to specify the duration of the new task (in seconds). Another task-switching operation is to stop execution of the current program and start a new program; the difference here is that the robot switches from one sequence of instructions to a different sequence of instructions, rather than just executing an atomic behavior. An operational requirement is that desired programs need to be numbered and known to the robot beforehand.

- Parameter reconfiguration: This is to instruct the robot to continue the current program with updated parameter values. This enables underwater missions to continue unimpeded (as discussed in Section \), without interrupting the current task or requiring the robot to be brought to the surface. Here, the requirement is that the tunable parameters need to be numbered and their choice of values need to be specified beforehand. The robot can also be instructed to take pictures (for some time) while executing the current program. This is important for underwater missions, as this facilitates visual logging as the robot executes a preset mission. Also, other sensory data can easily be logged in the same mechanism through a simple extension of the command triggered by a gesture.

The proposed framework supports a number of task switching and parameter reconfiguration instructions, which can be extended to accommodate more instructions by simply changing or appending a user-editable configuration file. The hand gesture-to-token mapping is carefully designed so that the robot formulates executable instructions only when intended by the diver. This is done by attributing specific hand gestures as sentinels (i.e., start- or end-tokens). Fig. 4 illustrates the gesture to atomic-instruction mapping used in our framework. Additional examples are shown in Fig. 5, where series of (start_token, instruction, end_token) tuples are mapped to corresponding sequences of gesture_tokens.

\section{B. Hand Gesture Recognition and Instruction Generation}

Any human-robot communication language must be able to recognize individual tokens robustly, independent of the modality used (e.g., aural, tactile or visual). In the proposed

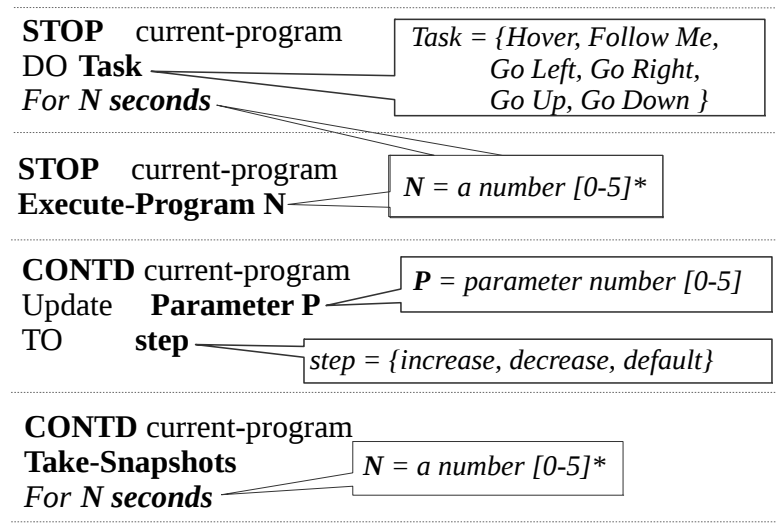

Fig. 3: Set of task switching and parameter reconfiguration instructions that are currently supported by our framework.

\begin{tabular}{|c|c|c|c|c|}
\hline \multirow[t]{2}{*}{ Instruction-token } & \multirow[t]{2}{*}{ Type } & \multicolumn{2}{|c|}{ Hand gestures } & \multirow{2}{*}{$\begin{array}{c}\text { Gesture-token } \\
\quad\{\text { left, right }\}\end{array}$} \\
\hline & & Left & Right & \\
\hline STOP current-program & Start-token & 8 & d & $\{0, \mathrm{ok}\}$ \\
\hline HOVER & Task & 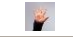 & 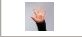 & $\{5,5\}$ \\
\hline FOLLOW me & Task & 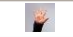 & 6 & $\{5,1\}$ \\
\hline Go LEFT & Task & 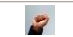 & 는 & $\{0$, left $\}$ \\
\hline Go RIGHT & Task & 3 & I & $\{0$, right $\}$ \\
\hline Go UP & Task & 8 & I & \{right, right $\}$ \\
\hline Go DOWN & Task & E & 늘 & $\{$ left, left $\}$ \\
\hline EXECUTE Program & Task & t & 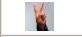 & $\{$ pic, 2$\}$ \\
\hline CONTD current-program & Start-token & 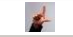 & 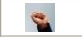 & $\{$ pic, 0$\}$ \\
\hline Take SNAPSHOT & Task & t & 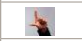 & $\{$ pic, pic\} \\
\hline $\mathrm{N}$ (number) & {$[0-5]^{*}$} & 6 & $8^{-}$ & $\{$ok, $0-5\}$ \\
\hline $\mathrm{P}$ (parameter number) & {$[0-5]^{*}$} & $3^{-1}$ & 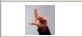 & $\{0-5$, pic $\}$ \\
\hline next_digit & indicator & t & $d$ & $\{$ pic, ok\} \\
\hline Increase & step & 8 & $y$ & \{right, pic\} \\
\hline Decrease & step & 8 & 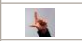 & $\{$ left, pic\} \\
\hline Default & step & 8 & $a$ & $\{$ ok, pic $\}$ \\
\hline GO & End-token & 6 & $d$ & $\{\mathrm{ok}, \mathrm{ok}\}$ \\
\hline
\end{tabular}

Fig. 4: Mapping of gesture-token to instruction-token used in our framework.

framework, the challenges lie in segmenting the hand gestures from the camera image, accurately recognizing the hand gestures, and then mapping the gestures to instructions. Fig. 6 illustrates the overall process, and the implementation details of each computational component of our framework are described in the following sections.

1) Region Selection: To detect gestures, the hand regions need to be cleanly extracted from the image, which can be challenging in underwater visual conditions which are often degraded. These regions are rectangular but vary in size as divers can be at different distances from the robot. One possible approach is to slide a rectangular window at multiple scales sequentially over the image and attempt hand detections; however, trying each possible rectangular region in such a brute-force fashion is not feasible due to computational and real-time operational constraints. Instead, the following approach is adopted:

1) First, the camera image (in RGB space) is blurred and thresholded (in HSV-space) for skin-color segmentation 


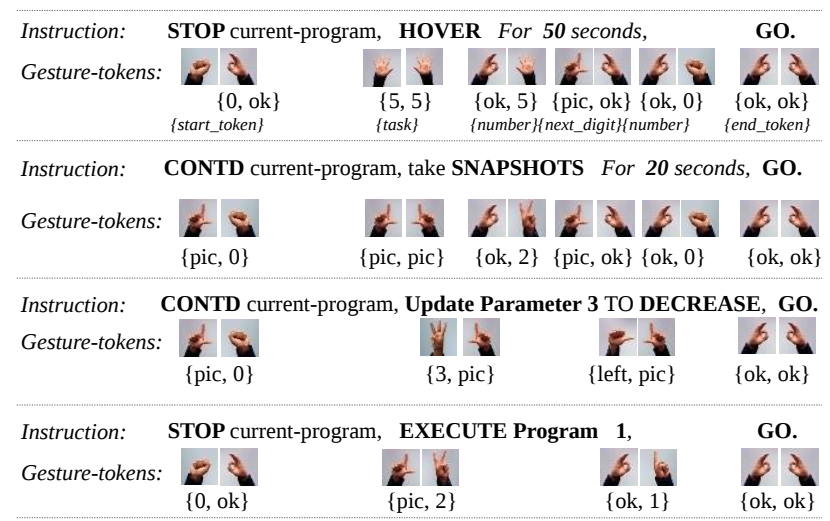

Fig. 5: Some examples of different combinations of hand gestures which are used to generate instructions (based on the gesture-instruction mapping shown in Fig. 47.

[16]. Here, we assume the diver performs gestures with bare hands; if the diver is to wear gloves, the color thresholding range in the HSV space needs to be adjusted accordingly.

2) Contours of the different segmented regions in the filtered image space are then extracted (see Fig. 7). Subsequently, different contour properties such as convex hull boundary and center, convexity defects, and important curvature points are extracted. We refer readers to [24] for details about properties and significance of these contour properties.

3) Next, outlier regions are rejected using cached information about the scale and location of hand gestures detected in the previous frame. This step is of course subject to availability of the cached information.

4) Finally, the hand contours of potential regions are matched with a bank of hand contours that are extracted from training data (one for each class of hand gestures as shown in the bottom row of Fig. 22. Final regions for left and right hand gestures are selected using the proximity values of the closest contour match [24]; i.e., the region that is most likely to contain a hand gesture is selected.

2) CNN Model for Gesture Recognition: Following region selection, cropped and resized image-patches of $32 \times 32$ are fed to a convolutional neural network (CNN) for gesture recognition. The detailed architecture of the model is illustrated in Fig. 9. Two convolutional layers are used for extracting and learning the spatial information within the images. Spatial down-sampling is done by max-pooling, while the normalization layer is used for scaling and reentering the data before feeding it to the next layer. The extracted feature vectors are then fed to fully connected layers to learn decision hyperplanes within the distribution of training data. Finally, a soft-max layer provides output probabilities for each class, given input data. Note that similar CNN models have been shown to perform well for small-scale (i.e., 10class classification) problems which are similar to ours.

The dimensions of each layer and the number of param- eters are specified in Fig. 9, the details about training and data-sets are provided in Section IV. The trained model is used for classifying hand gestures on $32 \times 32$ image patches provided by the region selection step. The classified gesturetokens are passed to a Finite-State Machine (FSM)-based gesture-to-instruction decoder, which we discuss next.

3) FSM-based Gesture to Instruction Decoder: An FSMbased deterministic model is used for efficient gesture-toinstruction mapping. As illustrated in Fig. 10, the transitions between instruction tokens are defined as functions of gesture tokens based on the rules defined in Fig. 4 Here, we impose an additional constraint that each gesture token has to be detected for 15 consecutive frames for the transition to be activated. This constraint adds robustness to missed or wrong classification for a particular gesture token. Additionally, it helps to discard noisy tokens which may be detected when the diver changes from one hand gesture to the next. Furthermore, since the mapping is one-to-one, it is highly unlikely that a wrong instruction will be generated even if the diver mistakenly performs some inaccurate gestures because there are no transition rules other than the correct ones at each state.

\section{EXPERIMENTAL Results}

We present the experimental results and discuss related implementation details of the proposed framework.

\section{A. Training the CNN Model}

The CNN model is implemented using TensorFlow [1] and trained on a Linux machine (CPU) over 35K RGB-images of hand gestures ( $3.5 \mathrm{~K}$ for each class). Training data contain $32 \times 32 \times 3$ images from both underwater and terrestrial environments. A few samples from the training images are shown in Fig. 2 and details about our CNN model are provided in Fig. 9. An additional $4 \mathrm{~K}$ images are used for validation and a separate $1 \mathrm{~K}$ images are used as a test-set.

It takes about two hours to train the model over 50 epochs after which it reaches a training accuracy of 0.997 . Once the network is trained, model parameters are saved, which are loaded later during testing to perform inference. Fig. 11 illustrates the learning behavior of the network in terms of training loss and classification accuracy. Maximum validation accuracy of 0.986 is achieved after 50 epochs of training, and the test-set accuracy is 0.969. Fig. 12 shows the confusion matrix based on test-set performance of the model.

\section{B. Performance Evaluation on Real-World Data}

We used an underwater drone (OpenROV 2.8 [2]) for our experiments in a closed-water (swimming pool) scenario. Sequences of hand gestures pertaining to different types of instructions are performed by three participants. In addition, synthetic test data is generated by augmenting different combinations of the recorded gesture sequences.

The test data is used to evaluate the performance of our framework, as demonstrated in Fig. 13. There are a total of 30 sets of image sequences in the test set (each image is 


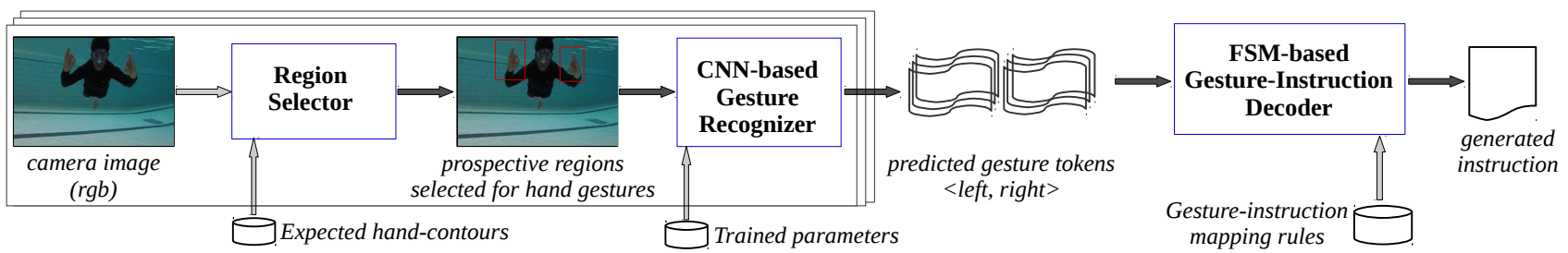

Fig. 6: Overview of the process to map hand gestures to instructions; the left half demonstrates the CNN-based recognition system, whereas the right half depicts the finite-state machine for robust mapping of gestures to instructions.

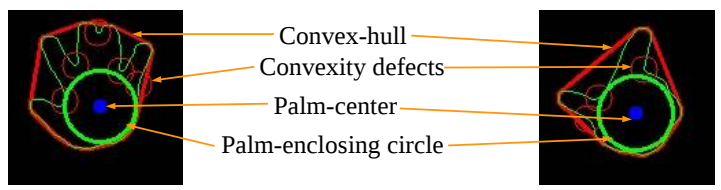

Fig. 7: Two examples of hand contours possessing different contour properties; the left image corresponds to gesture ' 5 ' while the right image corresponds to gesture 'pic'.

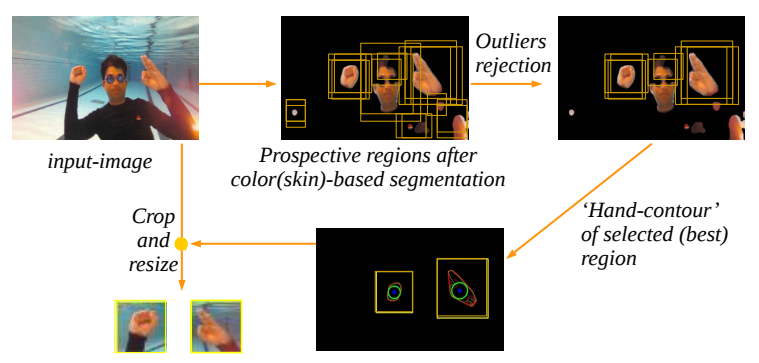

Fig. 8: Outline of the region selection mechanism of our framework: First, the (skin)color-based segmentation is performed to get potential regions for hand gestures; then, outliers are discarded based on cached information about the previous locations of the hands.

$640 \times 480 \times 3$ ). An additional 20 sets of test data are collected on land to inspect the performance in noise-free visual conditions. Table [ summarizes the performance of our framework for both classes of test data. We find that the overall accuracy of the framework mostly depends on region selection; that is, once the hand gestures are correctly segmented out, gesture recognition and gesture-to-instruction mapping demonstrate a high-degree of accuracy. As demonstrated by the bottom row of Table I] our framework successfully decoded all instructions from the noise-free terrestrial data even though gesture recognition accuracy was not perfect (i.e., 0.945). This is due to the robust FSM-based gesture-to-instruction mapping that ensures the following transition rules:

- State transitions are activated only if the corresponding gesture tokens are detected for 15 consecutive frames.

- There are no transition rules (to other states) for incorrect gesture tokens.

Consequently, an incorrect recognition has to happen 15 consecutive frames to generate an incorrect instruction, which is highly unlikely. However, in challenging visual conditions, region selection often fails to segment out the hand gestures correctly, which causes the overall process to fail. As the first row of Table $\mathbb{1}$ suggests, our framework fails in 6 test cases out of 30 . We inspected the failed cases and found the following issues:

- Surface reflection and air bubbles often cause problems for the region selector. Although surface reflection is not common in deep water, suspended particles and limited visibility will be additional challenges in deep openwater scenarios.

- In some cases, the diver's hand(s) appeared in front of his face or only partially appeared in the field-ofview. In these cases, not all of the hand(s) appeared in the selected region which eventually caused the gesture recognizer to detect ' 1 's as ' 0 's, or 'pic's as ' 1 's, etc.

TABLE I: Performance evaluation of our framework based on real-world data.

\begin{tabular}{|c|c||c|c|}
\hline $\begin{array}{c}\text { Operating } \\
\text { Medium }\end{array}$ & $\begin{array}{c}\text { Total \# of } \\
\text { Instructions (Gestures) }\end{array}$ & $\begin{array}{c}\text { Successfully } \\
\text { Decoded }\end{array}$ & $\begin{array}{c}\text { Accuracy } \\
(\%)\end{array}$ \\
\hline \hline Underwater & $30(162)$ & $24(128)$ & $80(78)$ \\
\hline Terrestrial & $20(132)$ & $20(121)$ & $100(94.5)$ \\
\hline
\end{tabular}

\section{Performance Evaluation through Gazebo Simulation}

We also performed simulation experiments on controlling an Aqua robot [6] based on instructions generated from sequence of hand gestures performed by a participant. The gesture sequences are captured through a webcam and the simulation is performed in Gazebo on the ROS Kinetic platform. As illustrated in Figure 14, gesture tokens are successfully decoded to control the robot. Although a noise-free simulation environment does not pose most challenges that are common in the real world, it does help set benchmarks for expected performance bounds and is useful in human interaction studies, which is described in the following section.

\section{Human Interaction Study}

We performed a human interaction study where the participants are introduced to our hand gesture based framework, the fiducial-based RoboChat framework [9], and the RoboChat-Gesture framework [23] where a set of discrete motions from a pair of fiducials are interpreted as gesturetokens. AprilTags [17] were used for the RoboChat trials to deliver commands.

A total of ten individuals participated in the study, who were grouped according to their familiarity to robot programming paradigms in the following manner: 


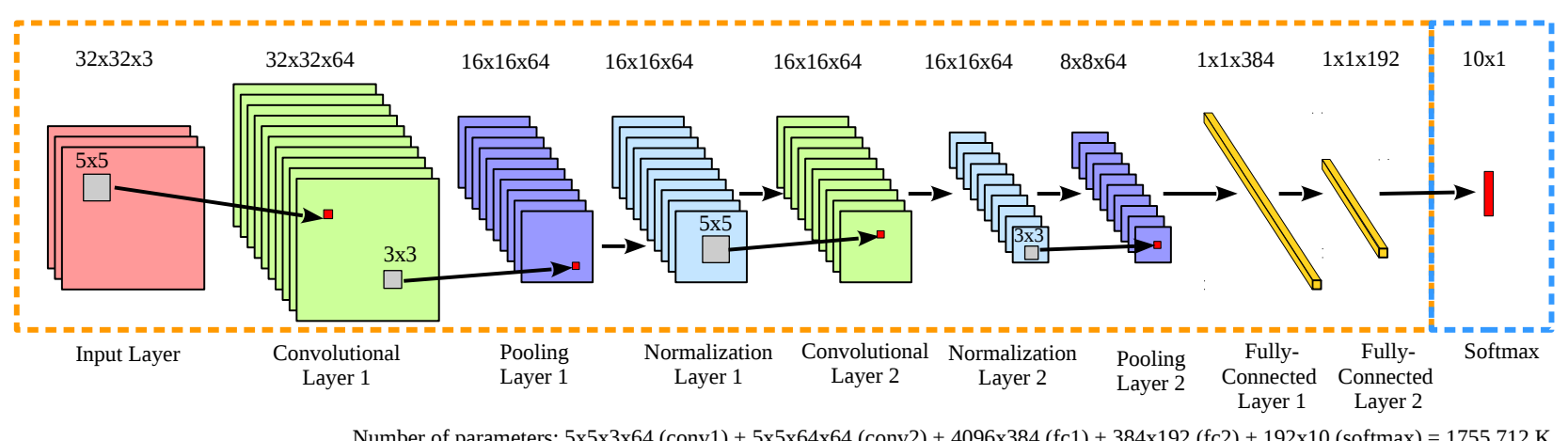

Fig. 9: Architecture of the CNN model used in our framework for hand gesture recognition.

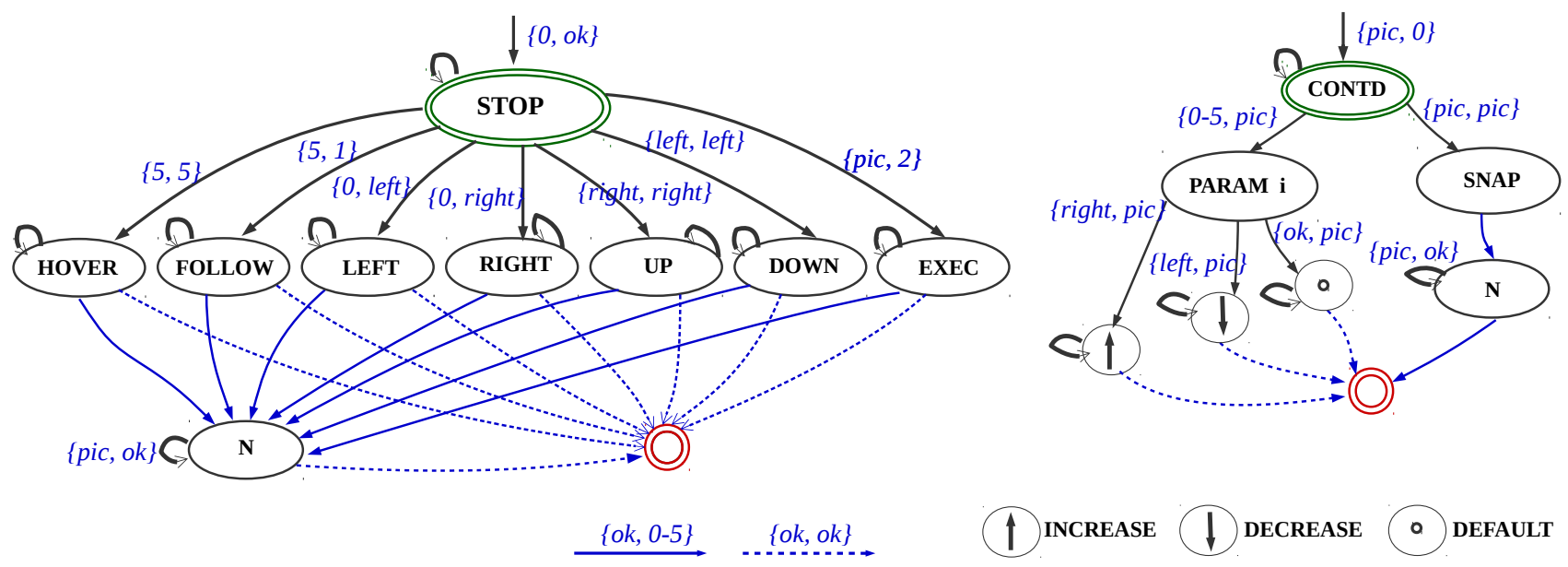

Fig. 10: FSM-based deterministic mapping of gestures-to-instructions (based on the rules defined in Fig. 4 .

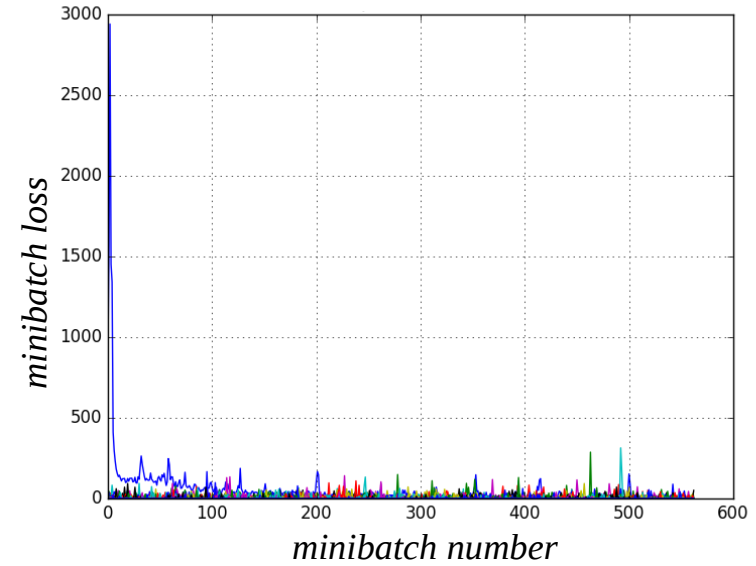

(a) Minibatch loss over 50 epochs

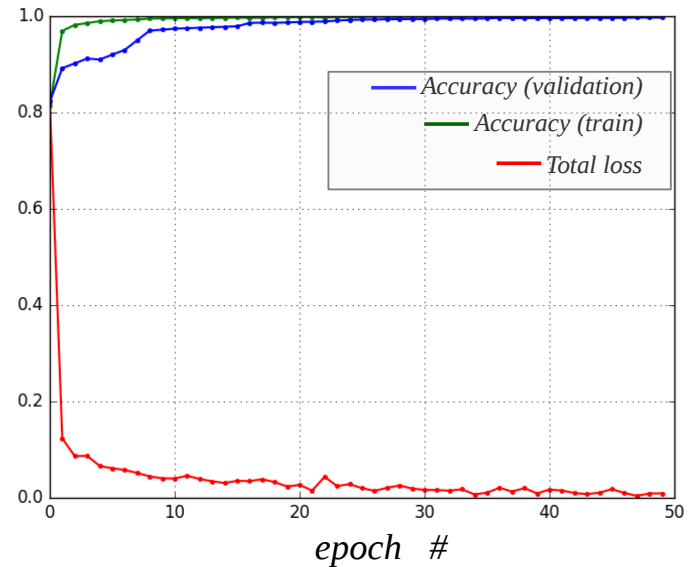

(b) Avg. total loss and accuracy per epoch

Fig. 11: Training error and accuracy of our CNN model illustrated in Fig. 9, the training and validation sets contain 35K and $4 \mathrm{~K}$ images, respectively; batch-size was set to 128 while the network was trained for 50 epochs.

- Beginner: participants who are unfamiliar with gesture/fiducial based robot programming (2 participants)

- Medium: participants who are familiar with ges- ture/fiducial based robot programming (7 participants)

- Expert: participants who are familiar and practicing these frameworks for some time (1 participant) 


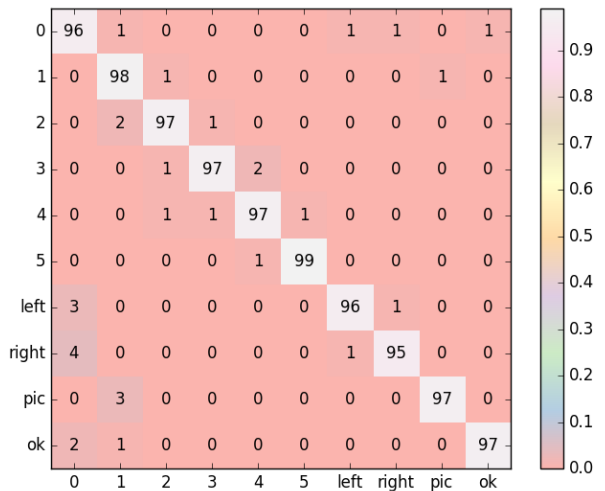

Fig. 12: Confusion matrix based on test-set performance.

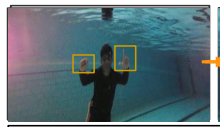

\{pic, 0$\}$

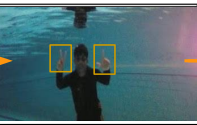

\{pic, 2\}

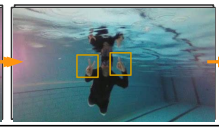

$\{$ ok, 2\} \{ok, ok $\}$

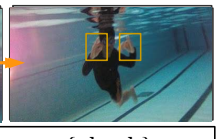

STOP current-program, EXECUTE Program 2, GO.

Fig. 13: Generation of instructions from a sequence of hand gestures using our model

This approach is similar to the one used by [23]. In the first set of trials, participants are asked to perform sequences of gestures to generate the following instructions (Fig. 5) in all three interaction paradigms:

1. STOP current-program, HOVER for 50 seconds, GO.

2. CONTD current-program, take SNAPSHOTS for 20 seconds, GO.

3. CONTD current-program, Update Parameter 3 to DECREASE, GO.

4. STOP current-program, EXECUTE Program 1, GO.

The second set of trials, participants had to program the robot with complex instructions and were given the following two scenarios:

a. The robot has to stop its current task and execute program 2 while taking snapshots, and

b. The robot has to take pictures for 50 seconds and then start following the user.

For all the experiments mentioned above, participants performed gestures with hands, AprilTags, and discrete motions with AprilTags. Correctness and the amount of time taken were recorded in each case. Fig. 15 shows the comparisons of average time taken to perform gestures for generating different types of instructions. Participants quickly adopted the hand gestures to instruction mapping and took significantly less time to finish programming compared to the other two alternatives. Specifically, participants found it inconvenient and time consuming to search through all the tags for each instruction token. On the other hand, although performing a set of discrete motions with only two AprilTags saves time, it was less intuitive to the participants. As a result, it still took a long time to formulate the correct gestures for complex instructions, as evident from Fig. 15

One interesting result is that the beginner users took less

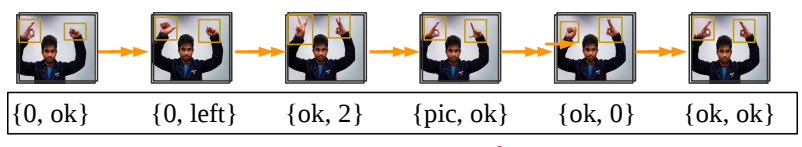

STOP current-program, go LEFT for 20 secs, GO.

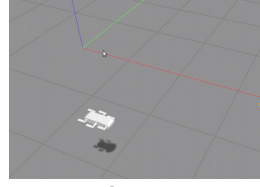

Aqua is hovering;

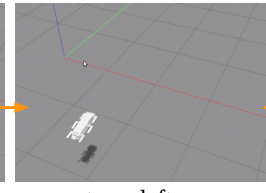

turns left;

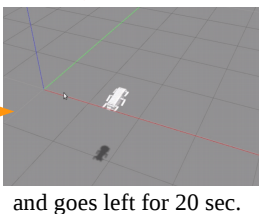

and goes left for $20 \mathrm{sec}$.
Fig. 14: Controlling an Aqua robot based on instructions generated from sequence of hand gestures performed by a person; the simulation is performed in Gazebo, on ROSkinetic platform

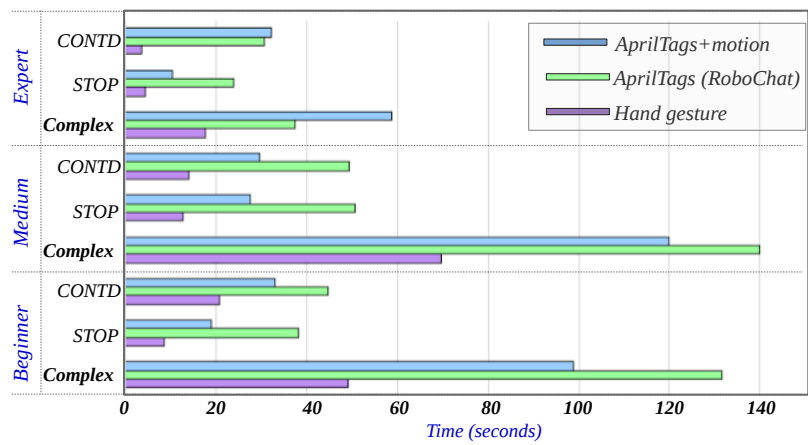

Fig. 15: Comparisons of average time taken to perform gestures for successfully generating different types of programs (STOP: instruction 1 and 4,CONTD: instruction 2 and 3, Complex: scenario $a$ and $b$ ) .

time to complete the instructions compared to medium users. This is probably due to the fact that unlike the beginner users, medium users were trying to intuitively interpret and learn the syntax while performing the gestures. However, as illustrated by Table $\Pi$, beginner users made more mistakes on average before completing an instruction successfully. The expert user performed all tasks on the first try, hence only a comparison for beginner and medium users is presented. Since there are no significant differences in the number of mistakes for any types of user, we conclude that simplicity, efficiency, and intuitiveness are the major advantages of our framework over the existing methods.

TABLE II: Average number of mistakes using [hand gesture, Robochat, AprilTtags with motion] for different users before correctly generating the instruction.

\begin{tabular}{|c|c||c|c|}
\hline $\begin{array}{c}\text { Instruction } \\
\text { Type }\end{array}$ & $\begin{array}{c}\text { Total \# of } \\
\text { Instructions (Gestures) }\end{array}$ & $\begin{array}{c}\text { Beginner } \\
\text { User }\end{array}$ & $\begin{array}{c}\text { Medium } \\
\text { User }\end{array}$ \\
\hline \hline STOP & $2(10)$ & {$[2,1,3]$} & {$[1,0,1]$} \\
\hline CONTD & $2(10)$ & {$[0,0,1]$} & {$[0,0,0]$} \\
\hline Complex & $2(16)$ & {$[2,3,7]$} & {$[2,2,3]$} \\
\hline
\end{tabular}

\section{CONCLusions And Future Work}

We present a hand gesture-based human-robot communication framework for underwater robots, where divers can use a set of intuitive and meaningful hand gestures to 
program new instructions or reconfigure existing program parameters for an accompanying robot on-the-fly. In the proposed framework, a CNN model provides accurate hand gesture recognition and an FSM-based deterministic model performs efficient gesture-to-instruction mapping. Accuracy and robustness of the framework is evaluated through extensive experiments, while a user interaction study is performed to evaluate the usability of the interface. Future work will investigate methods to accommodate a larger vocabulary of instructions and useful features, such as control-flow tokens, while maintaining simplicity and robustness of the approach. In addition, work will focus on designing an improved and more robust region selector. A complete evaluation of the interaction framework through open-water trials is the immediate next step.

\section{REFERENCES}

[1] Martín Abadi, Ashish Agarwal, Paul Barham, Eugene Brevdo, Zhifeng Chen, Craig Citro, Greg S Corrado, Andy Davis, Jeffrey Dean, Matthieu Devin, et al. Tensorflow: Large-scale machine learning on heterogeneous distributed systems. arXiv preprint arXiv:1603.04467, 2016.

[2] OPENROV CA. OpenROV Underwater Drone 2.8. https://www . openrov.com/products/openrov28/ Accessed: 8-30-2017.

[3] Sunjie Chen, Hongbin Ma, Chenguang Yang, and Mengyin Fu. Hand gesture based robot control system using leap motion. In International Conference on Intelligent Robotics and Applications, pages 581-591. Springer, 2015.

[4] D Chiarella, M Bibuli, G Bruzzone, M Caccia, A Ranieri, E Zereik, L Marconi, and P Cutugno. Gesture-based language for diver-robot underwater interaction. In OCEANS 2015-Genova, pages 1-9. IEEE, 2015.

[5] Enrique Coronado, Jessica Villalobos, Barbara Bruno, and Fulvio Mastrogiovanni. Gesture-based robot control: Design challenges and evaluation with humans. In 2017 IEEE International Conference on Robotics and Automation (ICRA), pages 2761-2767. IEEE, 2017.

[6] Gregory Dudek, Philippe Giguere, Chris Prahacs, Shane Saunderson, Junaed Sattar, Luz-Abril Torres-Mendez, Michael Jenkin, Andrew German, Andrew Hogue, Arlene Ripsman, et al. Aqua: An amphibious autonomous robot. Computer, 40(1), 2007.

[7] Gregory Dudek, Philippe Giguere, and Junaed Sattar. Sensor-based behavior control for an autonomous underwater vehicle. In Experimental Robotics, pages 267-276. Springer, 2008.

[8] Gregory Dudek, Michael Jenkin, Chris Prahacs, Andrew Hogue, Junaed Sattar, Philippe Giguere, Andrew German, Hui Liu, Shane Saunderson, Arlene Ripsman, et al. A visually guided swimming robot. In 2005 IEEE/RSJ International Conference on Intelligent Robots and Systems (IROS), pages 3604-3609. IEEE, 2005.

[9] Gregory Dudek, Junaed Sattar, and Anqi Xu. A visual language for robot control and programming: A human-interface study. In 2007 IEEE International Conference on Robotics and Automation (ICRA), pages 2507-2513. IEEE, 2007.

[10] Cameron Fabbri, Md Jahidul Islam, and Junaed Sattar. Enhancing underwater imagery using generative adversarial networks. 2018.

[11] Mark Fiala. ARTag, a fiducial marker system using digital techniques. In 2005 IEEE Computer Society Conference on Computer Vision and Pattern Recognition (CVPR), volume 2, pages 590-596. IEEE, 2005.

[12] Md Jahidul Islam and Junaed Sattar. Mixed-domain biological motion tracking for underwater human-robot interaction. In 2017 IEEE International Conference on Robotics and Automation (ICRA), pages 4457-4464. IEEE, 2017.

[13] Pavlo Molchanov, Shalini Gupta, Kihwan Kim, and Jan Kautz. Hand gesture recognition with 3D convolutional neural networks. In Proceedings of 2015 IEEE conference on computer vision and pattern recognition workshops, pages 1-7, 2015.

[14] Jawad Nagi, Frederick Ducatelle, Gianni A Di Caro, Dan Cireşan, Ueli Meier, Alessandro Giusti, Farrukh Nagi, Jürgen Schmidhuber, and Luca Maria Gambardella. Max-pooling convolutional neural networks for vision-based hand gesture recognition. In 2011 IEEE International
Conference on Signal and Image Processing Applications (ICSIPA), pages 342-347. IEEE, 2011.

[15] Natalia Neverova, Christian Wolf, Graham W Taylor, and Florian Nebout. Multi-scale deep learning for gesture detection and localization. In Workshop at the European conference on computer vision, pages 474-490. Springer, 2014.

[16] VA Oliveira and A Conci. Skin detection using HSV color space. In H. Pedrini, \& J. Marques de Carvalho, Workshops of Sibgrapi, pages $1-2,2009$.

[17] Edwin Olson. AprilTag: A robust and flexible visual fiducial system. In Robotics and Automation (ICRA), 2011 IEEE International Conference on, pages 3400-3407. IEEE, 2011.

[18] Junaed Sattar, Eric Bourque, Philippe Giguere, and Gregory Dudek. Fourier tags: Smoothly degradable fiducial markers for use in humanrobot interaction. In 2007 Fourth Canadian Conference on Computer and Robot Vision (CRV), pages 165-174. IEEE, 2007.

[19] Florian Shkurti, Wei-Di Chang, Peter Henderson, Md Jahidul Islam, Juan Camilo Gamboa Higuera, Jimmy Li, Travis Manderson, Anqi Xu, Gregory Dudek, and Junaed Sattar. Underwater multi-robot convoying using visual tracking by detection. In 2017 IEEE/RSJ International Conference on Intelligent Robots and Systems (IROS). IEEE, 2017.

[20] Marjorie Skubic, Dennis Perzanowski, Samuel Blisard, Alan Schultz, William Adams, Magda Bugajska, and Derek Brock. Spatial language for human-robot dialogs. IEEE Transactions on Systems, Man, and Cybernetics, Part C (Applications and Reviews), 34(2):154-167, 2004.

[21] Stefan Waldherr, Roseli Romero, and Sebastian Thrun. A gesture based interface for human-robot interaction. Autonomous Robots, 9(2):151-173, 2000.

[22] Michael T Wolf, Christopher Assad, Matthew T Vernacchia, Joshua Fromm, and Henna L Jethani. Gesture-based robot control with variable autonomy from the JPL BioSleeve. In 2013 IEEE International Conference on Robotics and Automation (ICRA), pages 1160-1165. IEEE, 2013.

[23] Anqi Xu, Gregory Dudek, and Junaed Sattar. A natural gesture interface for operating robotic systems. In 2008 IEEE International Conference on Robotics and Automation (ICRA), pages 3557-3563. IEEE, 2008

[24] Hui-Shyong Yeo, Byung-Gook Lee, and Hyotaek Lim. Hand tracking and gesture recognition system for human-computer interaction using low-cost hardware. Multimedia Tools and Applications, 74(8):2687$2715,2015$. 